\title{
NASA unveils exoplanet haul
}

Findings from the now-disabled Kepler probe have been validated, quadrupling its booty of extrasolar worlds.

\section{Ron Cowen}

26 February 2014

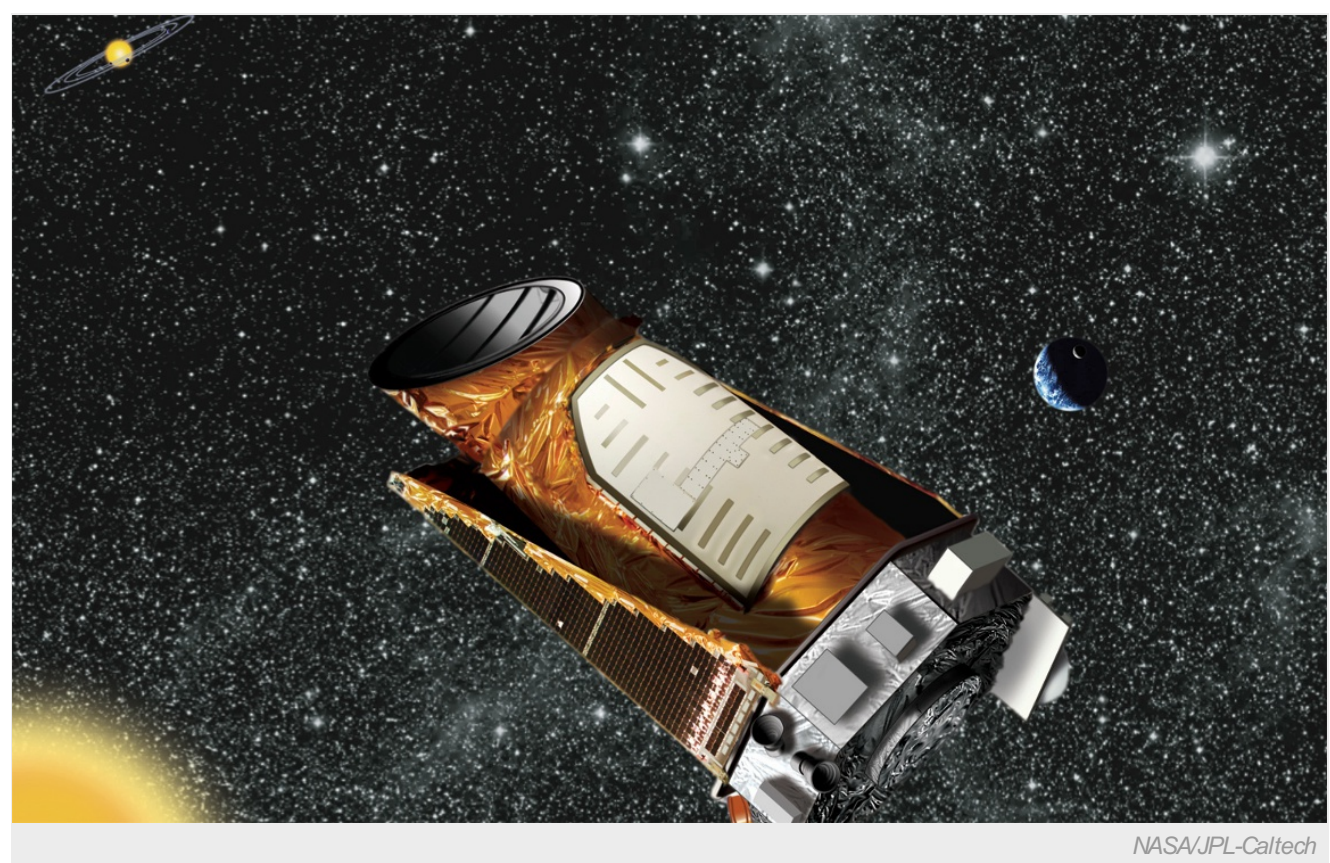

Kepler's discoveries have bumped the number of known planets beyond the Solar System to 1,750.

Astronomers announced today that 715 exoplanet candidates found by NASA's Kepler spacecraft have been confirmed as planets. The bonanza nearly quadruples the number of already confirmed alien worlds found by Kepler, and raises the total number of known planets beyond the Solar System from 1,035 to 1,750 .

The findings provide a mother lode of new data for studies of the statistical properties of exoplanets, and may bode well for finding a planet just like home, says Kepler researcher Jack Lissauer of NASA's Ames Research Center in Moffett Field, California.

The orbs all belong to multiple-planet systems — something that helps to confirm the nature of the candidate exoplanets quickly, notes Lissauer. He and his colleague Jason Rowe, of the SETI Institute in Mountain View, California, describe their analyses in two papers in press at the Astrophysical Journal ${ }^{1,2}$.

Kepler, which ended its main planet-hunting mission in May 2013 after a mechanical failure, searched for planets by detecting the tiny dip in starlight each time an orbiting body transited, or crossed in front of, its parent star. But researchers must account for confounding signals, such as a drop in illumination when one star eclipses another, which can mimic the signature of a passing planet. In earlier work ${ }^{3}$, Rowe, Lissauer and their colleagues showed that candidate planets orbiting a star in the company of one or more other planets have only a $0.2 \%$ chance of being spurious, compared with a $10 \%$ rate for candidate planets orbiting solo.

"We can tell that most of the multiple-planet candidates are good, with only a handful of problem cases," says Rowe. With that knowledge, he, Lissauer and their colleagues realized that they would not have to do much follow-up work to confirm Kepler candidates that were members of multiple-planet systems and could quickly process large batches of data.

\section{Habitable zones}

The new roster of planets, inferred from data collected by Kepler between 2009 and 2011, suggests that multiple-planet systems are relatively common among the roughly 150,000 stars that the craft continuously monitored. The latest finds include four planets in the habitable zone of their stars - the region that could be just the right temperature for water to be liquid — but all four orbs are about twice as large as Earth, says Rowe. The 715 planets are distributed among 305 multiplanetary systems that feature nearly-circular 
orbits such as those of the Solar System's terrestrial planets, but are much more compact.

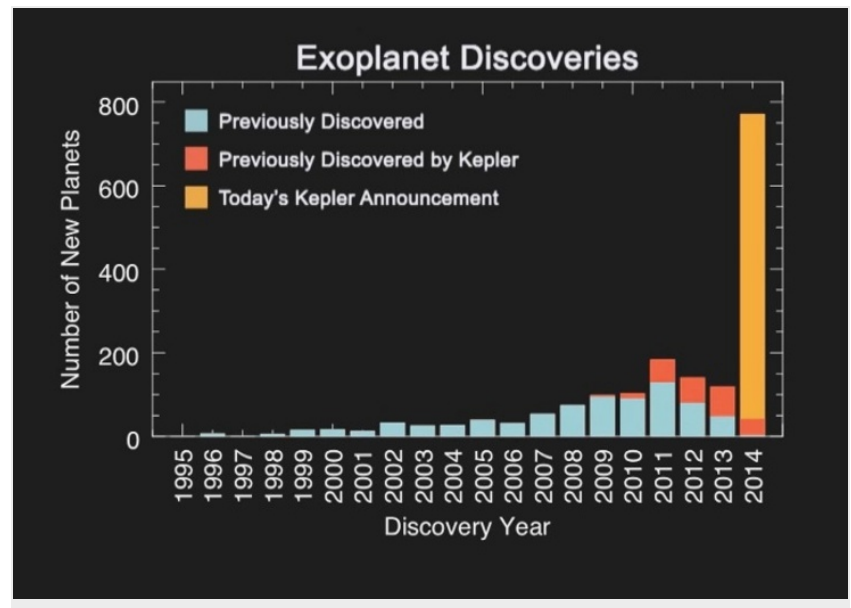

NASA Ames/SETVJ Rowe

Although many of the exoplanets are larger than Earth, these planetary systems "are scaled-down versions of our inner Solar System, giving us the chance to look at the Kepler data set and see hints of ourselves - systems that remind us of home", says Rowe.

Given that transits of short-period planets larger than Earth are easiest for Kepler to detect, "these new results continue to strengthen the case that Earth-like planets appear to be commonplace in our Universe, even if they remain devilishly difficult to discover and characterize", says planet hunter Alan Boss of the Carnegie Institution for Science in Washington DC, who was not part of the studies.

Astronomers said today that Kepler has found another 715 confirmed exoplanets.

Sara Seager, an astrophysicist at the Massachusetts Institute of Technology in Cambridge, says the planetary windfall reinforces Kepler's major finding: "Small planets are extremely common in our Galaxy."

If an Earth-sized planet orbiting a Sun-like star is hiding in a multiple-planet system, it should be easier to detect than if it is orbiting solo, says Lissauer. Such systems could be targets for NASA's Transiting Exoplanet Survey Satellite, scheduled for launch in 2017.

Nature | doi:10.1038/nature.2014.14779

\section{References}

1. Lissauer, J. J. et al. Astrophys. J. in the press (2014).

2. Rowe, J. F. et al. Astrophys. J. in the press (2014).

3. Lissauer, J. J. et al. Astrophys. J. 750, 112 (2012). 